\title{
छூ \\ Correlating scanning tunneling spectroscopy with the electrical resistivity of Al-based quasicrystals and approximants
}

\author{
Ruben Mäder, ${ }^{1,2}$ Roland Widmer, ${ }^{1}$ Pierangelo Gröning, ${ }^{1}$ Walter Steurer, ${ }^{2}$ and Oliver Gröning ${ }^{1}$ \\ ${ }^{1}$ Empa, Swiss Federal Laboratories for Materials Science and Technology, nanotech@surfaces Laboratory, Überlandstrasse 129, \\ CH-8600 Dübendorf, Switzerland \\ ${ }^{2}$ ETH Zürich, Laboratory of Crystallography, Department of Materials, Wolfgang-Pauli-Str. 10, CH-8093 Zürich, Switzerland
}

(Received 3 December 2012; published 19 February 2013)

\begin{abstract}
We present detailed low-temperature scanning tunneling spectroscopy (STS) investigations of the local electronic density of states (DOS) on the fivefold and twofold $i$ - $\mathrm{Al}_{70} \mathrm{Pd}_{21} \mathrm{Mn}_{9}$; pseudo tenfold $\xi^{\prime}-\mathrm{Al}_{72} \mathrm{Pd}_{25} \mathrm{Mn}_{3}$; tenfold $d-\mathrm{Al}_{77} \mathrm{Ni}_{17} \mathrm{Co}_{6}$; twofold-(12110) $d-\mathrm{Al}_{72.9} \mathrm{Ni}_{10.4} \mathrm{Co}_{16.7}$; (100) $\mathrm{Y}_{-} \mathrm{Al}_{75.8} \mathrm{Ni}_{2.1} \mathrm{Co}_{22.1}$; and (111) $\mathrm{Al}$ surface in the range of $+/-1 \mathrm{eV}$ around the Fermi energy. All these quasicrystals and approximants exhibit the theoretically predicted electronic pseudogap. We observe no evidence for a correlation between the depth of this pseudogap, as measured by STS, and the remarkably high electrical resistivity of the investigated quasicrystals and approximants. A parametrization of the spatial variability of STS spectra is introduced as a measure of the magnitude of the electron localization for a given system. The resulting $\hat{S}$ parameter is found to be proportional to the square root of the electrical resistivity. This finding supports the validity of interpreting spatial variations of fine-structure features in the STS spectra of Al-based quasicrystals and approximants in terms of electron localization.
\end{abstract}

DOI: 10.1103/PhysRevB.87.075425

PACS number(s): 71.23.Ft, 68.37.Ef, 68.35.B-

\section{INTRODUCTION}

Quasicrystals (QC) are intermetallics exhibiting rotational symmetries that are incompatible with three-dimensional translational invariance but showing a very high degree of quasiperiodic atomic long-range order. ${ }^{1,2}$ Along with their quasiperiodic crystal structures, QC are well known to exhibit physical properties markedly different from those of their constituent elements. ${ }^{3}$ They show comparatively high electrical resistivities with predominantly negative temperature coefficients, ${ }^{4,5}$ low thermal conductivities, ${ }^{3}$ low friction coefficients, ${ }^{6}$ and low surface energies. ${ }^{3}$ Much effort has been devoted to understand the influence of the quasiperiodic order on the remarkable physical properties of QC. ${ }^{7}$ In the case of decagonal QC, it is possible to directly compare properties along the quasiperiodic with those of the periodic direction. This is made possible by the fact that the decagonal QC structure can be geometrically described as a periodic stacking of quasiperiodically ordered planes. ${ }^{8}$ Furthermore, the QC phase can be compared to its related approximant phases, which are alloys of similar composition and local atomic configuration but being periodic. Investigations of the electrical conductivity of the $d-\mathrm{Al}-\mathrm{Ni}-\mathrm{Co} \mathrm{QC}$ and the Y-Al-Ni-Co approximant show pronounced directional anisotropies. ${ }^{9-12}$ Both systems show a distinct asymmetry of the electrical resistivity along the quasiperiodic and periodic directions of the $d$-Al-Ni-Co QC (Ref. 4) $\left(\rho_{A} / \rho_{P} \approx 9\right)$ and between the corresponding directions of the Y-Al-Ni-Co approximant ${ }^{10}\left(\rho_{a} / \rho_{b} \approx 3.2\right.$ and $\left.\rho_{c} / \rho_{b} \approx 2.5\right)$. From these results, Smontara et al. concluded $^{10}$ that the long-range quasiperiodic order is at best of marginal importance for the anisotropy, which, according to them, originates from the complex local atomic structure. Evidently, the physical origin of the observed anisotropies is rooted in the electronic structure of the corresponding solids. From a general point of view, the valence electronic structure of aluminum-based QC and approximants, which are the subject of this study, is characterized by two features: first by a pseudogap at the Fermi energy $\left(E_{F}\right)$ with a width of typically one $\mathrm{eV},{ }^{13-29}$ which is attributed to the Hume-Rothery (HR) stabilization ${ }^{29}$ mechanism or to orbital hybridization; ${ }^{13}$ second by a collection of localized states around $E_{F}$ giving rise to a rich fine structure of peaks and pseudogaps in the density of states (DOS), ${ }^{17,30-41}$ which has been called "spiky" density of states for QC.

The pseudogap ${ }^{13,22}$ results either from Fermi-surface pseudo-Brillouin zone interactions, which lead to a depletion of electrons at $E_{F}$ HR mechanism, or by a hybridization of electron bands crossing each other at $E_{F}$. Both mechanisms stabilize the system by lowering its electronic energy. It has been theoretically ${ }^{17,20,21,23-26}$ predicted as well as experimentally ${ }^{14,15,18,19,28}$ observed for many QC and approximant systems. The influence of the pseudogap on the electronic transport, however, is not fully understood yet. ${ }^{14,16,27}$ Several ultraviolet photoemission spectroscopy (UPS) studies on icosahedral and decagonal QC could not correlate the dramatic change (up to two orders of magnitudes) of the electrical resistivity ${ }^{14,27}$ with the reduction of the DOS at $E_{F}$. On the other hand, the investigations of the electronic specific heat by Mizutani ${ }^{16}$ showed a very good correlation with the electrical resistivity. As indicated before, in addition to the pseudogap, one needs to consider localization of states at $E_{F}$ and the following question arises: What is the influence of this second characteristic of QC and approximants on the electronic transport $^{16,42}$ ?

The presence of a spiky DOS as a result of localization in QC and approximant is still much under debate theoretically ${ }^{17,30}$ as well as experimentally. ${ }^{14,32,33,36}$ With its high spatial and energy resolution, scanning tunneling spectroscopy (STS) experiments ${ }^{31,37,43}$ recently confirmed the theoretically predicted spiky character of the local DOS (LDOS). It has been shown ${ }^{31,37}$ that the spiky features in the LDOS are rapidly smoothed out by averaging over a surface area of a few tens of $\mathrm{nm}^{2}$. The origin of a spiky feature in the 
DOS has been attributed by Trambly de Laissardière et al. ${ }^{13}$ to localization of electronic states by complex atomic structures leading to so-called "cluster virtual bound states." This results in a very low velocity of corresponding charge carriers (and thus a high effective electron mass), which can explain the high resistivity of QC and approximant. ${ }^{42}$

In this work, our discussion considers both characteristics of the DOS structure by analyzing high-resolution STS results obtained on atomically flat surfaces. The investigated samples belong to two groups of aluminum-based QC and approximants: the $i$-Al-Pd-Mn and $d$-Al-Ni-Co groups. For a direct comparison with the main constituent element, we also measured the $\mathrm{Al}(111)$ surface as a reference. We characterized the pseudogap around the $E_{F}$ for all samples, where a detailed discussion of the normalization procedure of STS spectra is given in order to obtain a most faithful recovery of the DOS. Then, the spiky features of the LDOS representing spatial and energetic localization are analyzed and compared to topography structures. Finally, a new parameter is introduced to quantify from the STS measurements the degree of spikiness in different systems, which correlates with the corresponding electrical resistivity.

\section{EXPERIMENTAL DETAILS}

In Table I, the investigated single-crystalline samples are listed with their nominal composition, surface orientation, and reference of the sample preparation procedure. The surfaces were prepared by sputter-annealing cycles to show a step-terrace morphology with characteristic surface structure motifs as detailed in the corresponding references. Scanning tunneling microscopy (STM) imaging and STS measurements using the lock-in technique were performed with an Omicron low-temperature STM at $5.3 \mathrm{~K}$ and at a base pressure below $5 \times 10^{-11}$ mbar using mechanically cut $\mathrm{Pt}_{80} / \mathrm{Ir}_{20}$ tips. Parameters for the lock-in STS measurements were a $10-20 \mathrm{mV}$ amplitude (RMS) of the ac-voltage signal at a frequency of $860 \mathrm{~Hz}$. Large sets $(>500)$ of $d I / d V$ spectra have been recorded by acquiring single spectra on equidistant grid positions during a topography scan. These STS grid measurements served as a basis to evaluate the spatial variability in the LDOS.

In order to recover the pseudogap structure from the STS data, an appropriate normalization procedure is required, which we discuss in the following. Within the semiclassical Wentzel, Kramers, and Brillouin (WKB) approximation,

TABLE I. Overview of the investigated surfaces and their sample preparation procedure reference.

\begin{tabular}{llc}
\hline \hline System & Surface orientation & $\begin{array}{c}\text { Reference of the } \\
\text { surface preparation }\end{array}$ \\
\hline$i-\mathrm{Al}_{70} \mathrm{Pd}_{21} \mathrm{Mn}_{9}$ & Fivefold & 31 \\
$i-\mathrm{Al}_{70} \mathrm{Pd}_{21} \mathrm{Mn}_{9}$ & Twofold & 31 \\
$\xi^{\prime}-\mathrm{Al}_{72} \mathrm{Pd}_{25} \mathrm{Mn}_{3}$ & Pseudo tenfold & 44 \\
$\mu-\mathrm{Al}_{4} \mathrm{Mn}$ & $(001)$ & 45 \\
$d-\mathrm{Al}_{77} \mathrm{Ni}_{17} \mathrm{Co}_{6}$ & Tenfold (00001) & 46 \\
$d-\mathrm{Al}_{72.9} \mathrm{Ni}_{10.4} \mathrm{Co}_{16.7}$ & Twofold (12110) & 47 \\
$\mathrm{Y}-\mathrm{Al}_{75.8} \mathrm{Ni}_{2.1} \mathrm{Co}_{22.1}$ & $(100)$ & 43 \\
$\mathrm{Al}$ & $(111)$ & 48,49 \\
\hline \hline
\end{tabular}

Tersoff and Hamann ${ }^{50}$ derived the well-known expression for the tunneling current in STM. This expression relates the tunneling current and therefore also the differential conductance to the sample LDOS. For sufficiently low temperatures (where the Fermi distribution can be regarded as step function), the tunneling current can be written as

$$
I=A \int_{0}^{e V} \rho_{s}(x, y, E) \rho_{t}(E-e V) T(E, e V, z) d E,
$$

where $A$ is a proportionality factor, $T(E, e V, z)$ is the tunneling transmission probability, $\rho_{s}(x, y, E)$ and $\rho_{t}(E)$ are the surface and tip DOS at the location $(x, y)$, respectively. With the approximation of a constant DOS at the tip, the tunneling current is a convolution of the tunneling transmission probability and the LDOS of the sample. The standard method to obtain the sample LDOS is to record at a given position the $d I / d V$ signal as a function of the bias voltage. These unprocessed $d I / d V$ spectra deviate from the true sample LDOS due to the changing tunneling transmission probability with sample bias. Therefore, the raw $d I / d V$ spectra have to be normalized to yield a reliable measurement of the sample LDOS. The most prominent normalization procedure for a weakly varying, nearly constant DOS is performed by $(d I / d V) /(I / V)$ as proposed by Feenstra et $a l^{51}$ and theoretically justified by Hamers. ${ }^{52}$ In the case of semiconductors, the $(d I / d V) /(I / V)$ normalization has been modified by adding a constant term to the current so that within the band gap the expression is still defined. ${ }^{53}$

The experimental $d I / d V$ spectra of QC and approximants show that the assumption of a nearly constant DOS around $E_{F}$ is not correct. Rather, a pronounced parabolic pseudogap centered at $E_{F}$ is observed. ${ }^{14,15,20,21,31,43}$ The simple $(d I / d V) /(I / V)$ normalization might hence be unsuited in this case. We have tested several normalization procedures by numerical simulations, namely, those proposed by Feenstra et al., ${ }^{51}$ Prietsch et al., ${ }^{53}$ and Ukraintsev. ${ }^{54}$ For these tests, we assumed a convex parabolic sample DOS superimposed with a rapidly varying, sinusoidal modulation to emulate the spikiness. The tip DOS is kept constant. Subsequently, a full tunneling current calculation based on Eq. (1) has been performed from which the $d I / d V$ spectrum is obtained. The normalized $d I / d V$ spectra are then compared to the original sample DOS. The best recovery of the sample DOS has been achieved using a slightly modified version of the fitting technique proposed by Ukraintsev. ${ }^{54}$ The measured $d I / d V$ spectra were fitted by the following function:

$$
F(V)=\left(a+b V^{2}\right) \exp \left\{-2 \Delta z\left[\frac{2 m_{e}}{\hbar^{2}}(\Phi-e V)\right]^{0.5}\right\}
$$

where the first part approximates the parabolic pseudogap and the second part accounts for the asymmetry induced by the tunneling transmission probability. The variable $V$ represents the sample bias, $e$ is the elementary charge, $a$ and $b$ are fitting parameters for the sample DOS, $\Delta z$ is the fitting parameter related to the tip-sample distance, and $\Phi$ is the mean work function of the sample and the tip which was set to $4.5 \mathrm{eV}$, which is a reasonable value for QC and approximant. ${ }^{55}$ The normalization consists then of dividing the measured $d I / d V$ 

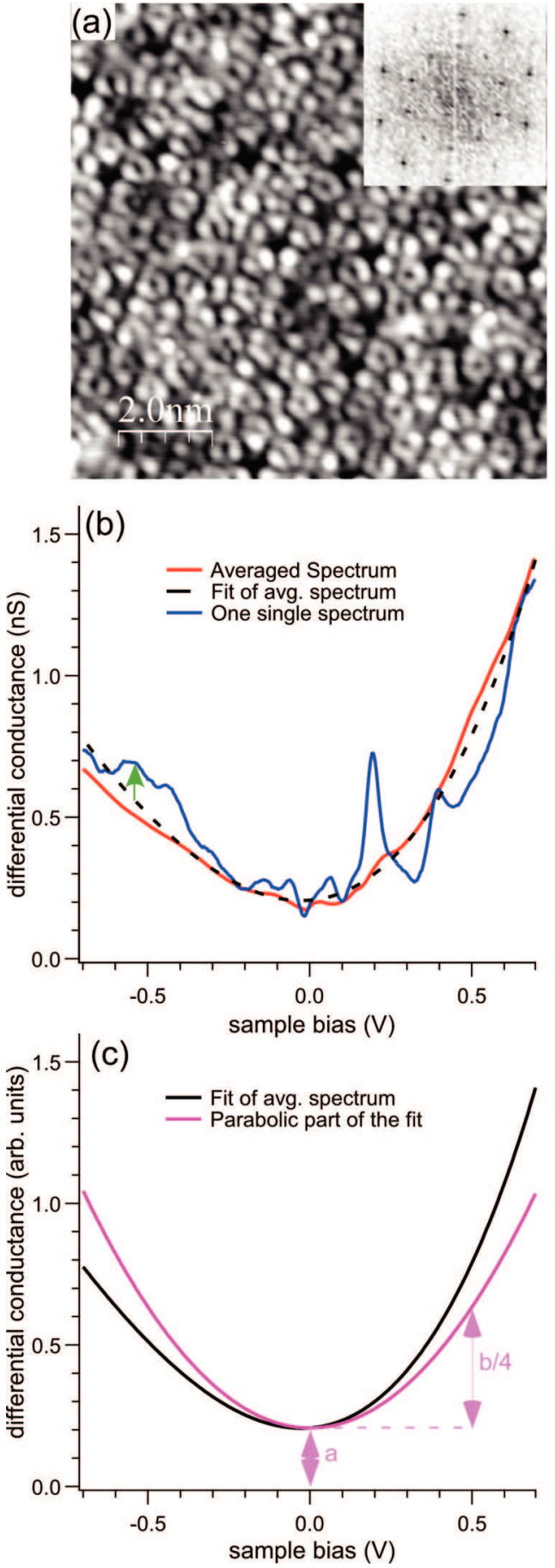

FIG. 1. (Color online) Illustration of the STS data analysis. (a) $10 \times 10 \mathrm{~nm}^{2}$ representative STM image of fivefold $i$-Al-Pd-Mn surface recorded at $5.3 \mathrm{~K}, \Delta z=0.5 \mathrm{~nm}, V_{T}=0.5 \mathrm{~V}, I_{T}=0.2 \mathrm{nA}$. Inset shows tenfold-symmetric pattern of the FFT image (inverted gray scale, $6.3 \times 6.3 \mathrm{~nm}^{-2}$ ) calculated from the presented surface. (b) Spectra of differential conductance $d I / d V$ vs bias voltage, with one representative single spectrum (blue line), the averaged spectrum (red line) calculated from 2380 single spectra, and the fit curve $F(V)$ of the averaged spectrum (dashed black line). Note the strong deviation of the single compared to the averaged spectrum, indicated by the vertical arrow. (c) Illustration of the STS normalization procedure. The removal of the contribution of the transmission function leads to a parabolic differential conductance structure. The parabolic part of the fit has been normalized to the value of the averaged spectrum at $E_{F}$. spectrum by the exponential term of Eq. (2) and by the parameter $a$.

The fitting procedure is exemplified in Fig. 1 for the STS results obtained on the fivefold $i-\mathrm{Al}_{70} \mathrm{Pd}_{21} \mathrm{Mn}_{9}$ surface. Figure 1(a) displays a typical STM topography image of the fivefold $i-\mathrm{Al}_{70} \mathrm{Pd}_{21} \mathrm{Mn}_{9}$ surface. The inset shows the fast Fourier transform (FFT) of this surface evidencing the quasiperiodic rotational symmetry by the appearance of rings with 10 spots. Within the size of a $10 \times 10 \mathrm{~nm}^{2}$ STM image, a STS grid measurement consisting of 2380 single spectra has been recorded. Figure 1(b) displays one $d I / d V$ single spectrum (blue curve) as well as the averaged spectrum (red curve) calculated from all 2380 single spectra. As reported by Widmer et al., ${ }^{31}$ the single $d I / d V$ spectra show a locally varying fine structure of peaks and valleys which vanishes when spectra are averaged already over small surface areas of a few $\mathrm{nm}^{2}$. The general parabolic appearance, however, is common to all single spectra recorded at different locations. Therefore, the fit function $F(V)$ [Eq. (2)] is applied on the smooth averaged $d I / d V$ spectrum. The dashed black line in Fig. 1(b) shows a good match of the resulting fit with the averaged $d I / d V$ spectrum. Figure 1(c) compares the resulting fit function according to Eq. (2) (black solid line) to the parabolic term only (pink line). This comparison evidences the asymmetry introduced by the tunneling transmission probability, which weights the LDOS stronger in the unoccupied DOS (positive sample biases) as compared to the occupied DOS (negative sample biases). The magnitude of the asymmetry depends on the tip-sample distance, where an increase of the tip-sample distance leads to a stronger asymmetry. This behavior is observed in the STS experiments as well as in our simulations.

In this sense, the parabolic part of the fit function can be understood as the pseudogap structure of the sample DOS and the exponential part corresponds to the asymmetry introduced by the tunneling transmission probability. The sample DOS obtained from the normalization is then characterized by the single parameter $b / a$ :

$$
\rho_{s} \propto(d I / d V)_{\mathrm{norm}} \approx 1+\frac{b}{a} V^{2} .
$$

Thus, the proportionality factor $b / a$ is a quantity for the depth and width of the pseudogap. The larger the $b / a$ ratio, the stronger is the curvature of the pseudogap with regard to its intensity at the $E_{F}$. The advantage of this description is that the pseudogap structure derived from $d I / d V$ spectra recorded on different surfaces and with different STS set points can be qualitatively compared to each other.

\section{RESULTS AND DISCUSSION}

The following discussion is divided in three sections: First, a characterization of the global $d I / d V$ structure in terms of the $b / a$ ratio is given. Then, the correlation between specific topographic features and the variation in the STS derived LDOS fine structure is discussed. Finally, the magnitudes of the deviations of single $d I / d V$ spectra compared to the averaged spectra are analyzed in order to quantify the local variability as a measure of the spikiness. This variability is then 
(a)

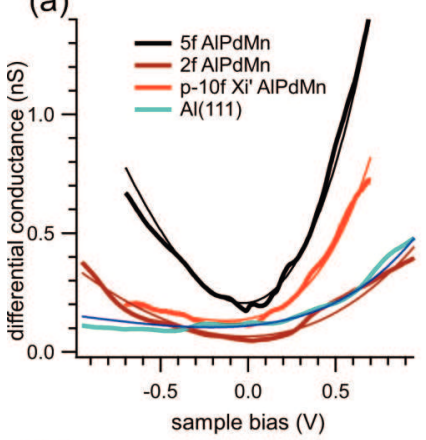

(b)

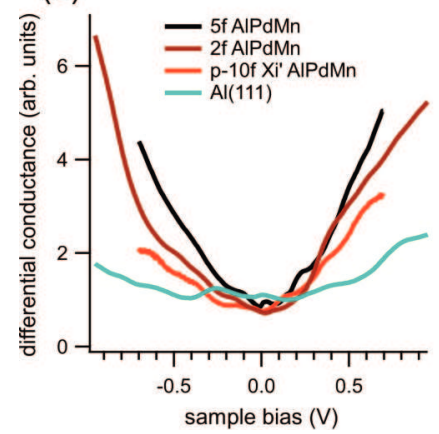

(c)

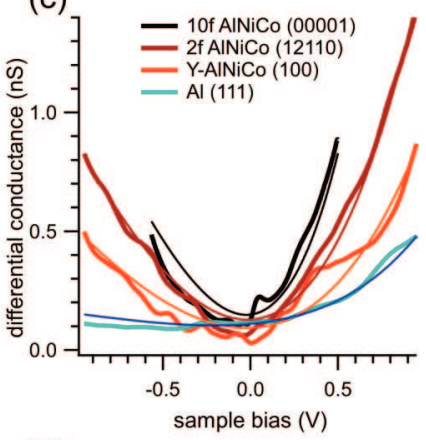

(d)

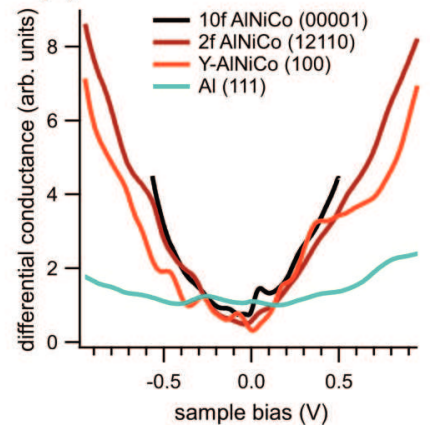

FIG. 2. (Color online) Overview of averaged [(a) and (c)] and normalized [(b) and (d)] differential conductance spectra vs bias voltage recorded by STS on the investigated surfaces. (a), (b) Spectra from fivefold and twofold $i$-Al-Pd-Mn QC, pseudo tenfold $\xi^{\prime}$-Al-Pd-Mn approximant, and $\mathrm{Al}(111)$ surfaces. (b), (c) Spectra from tenfold and twofold $d$-Al-Ni-Co QC, (100) Y-Al-Ni-Co approximant, and $\mathrm{Al}(111)$ surfaces.

compared to the electrical resistivities of the corresponding system.

\section{A. Analysis of the averaged differential conductance structure}

Figure 2 presents an overview of the averaged $d I / d V$ spectra (thick solid lines) as well as of the corresponding fits according to Eq. (2) (thin solid lines) of the Al-Pd-Mn alloys [Fig. 2(a)] and of the Al-Ni-Co alloys [Fig. 2(c)]. In all graphs of Fig. 2, the characteristics of the $\mathrm{Al}(111)$ surface are added for reference. The averaged $d I / d V$ spectra of all surfaces show a parabolic shape and can be well fitted by Eq. (2), where the $\Delta z$ parameter, related to the tip-sample distance, was always in the physically meaningful range of 0.25 to $0.5 \mathrm{~nm}$.

In Figs. 2(a) and 2(c), one can observe that the nonnormalized spectra exhibit different curvatures and offsets. For the spectra of the fivefold and the twofold $i$-Al-Pd-Mn QC surfaces shown in Fig. 2(a), these differences are especially apparent. Although due to the icosahedral structure, ${ }^{56}$ one would expect similar characteristics for the two surfaces. The origin of these differences can be attributed to a different $\Delta z$ parameter due to different set-point parameters (see Table I). This comparison shows that without normalization, $d I / d V$ spectra recorded under different conditions are hardly comparable.

On the other hand, the shapes of the normalized $d I / d V$ spectra of the fivefold and the twofold $i$-Al-Pd-Mn surface are very much alike and they are clearly different from the one

of the $\mathrm{Al}(111)$ surface. This shows the need of applying the normalization procedure described in the experimental section to allow a direct comparison. Table II summarizes the values of the $b / a$ ratios, the electrical resistivity, and the stabilization parameter used before opening the feedback loop for recording $d I / d V$ spectra for the seven investigated surfaces. A trend for the $b / a$ ratio to increase going from $\mathrm{Al}(111)$ via the approximants to the QC surfaces is observed. However, the most important observation is that the $b / a$ ratio is three to seven times smaller for the $\mathrm{Al}(111)$ as compared to the QC and approximant surfaces.

This finding supports our interpretation that the parabolic shape of the $d I / d V$ spectra on QC and approximants originates from a reduced DOS at $E_{F}$, which is reminiscent of the pseudogap. Other reports ${ }^{33-35,60}$ of tunneling spectroscopy on quasicrystalline surfaces assigned the parabolic structure in this large sample bias range of $+/-1 \mathrm{eV}$ to a purely tunneling transmission background. The argument that the observed curvature is indeed related to a pseudogap structure is further supported by the following: The $b / a$ ratio for QC and approximants is significantly larger compared with $\mathrm{Al}(111)$ regardless of the magnitude of the tunneling resistance $R_{T}$.

In general, we observe that the $b / a$ ratio is larger for QC (average 7.6) as compared to the approximants (average 5.6). A similar trend was observed by Belin-Ferré ${ }^{18}$ in the $\mathrm{Al}-\mathrm{Cu}-\mathrm{Fe}$ system as well as by Tamura et al. ${ }^{25}$ in the $\mathrm{Cd}-\mathrm{Yb}$ system by means of ultraviolet photoemission spectroscopy (UPS). In the latter case, the depth of the pseudogap near $E_{F}$ is reduced by factor of 1.7 for the $\mathrm{Cd}_{6} \mathrm{Yb}$ approximant as compared to the $i-\mathrm{Cd}_{5.7} \mathrm{Yb} \mathrm{QC}$.

It is very apparent that the $b / a$ ratios do not correlate with the reported values of the low-temperature electrical resistivities as shown in Table II. This observation is remarkable and is again in line with results from photoemission spectroscopy. Stadnik et al. ${ }^{14}$ could also not correlate the values of the depth of the UPS measured pseudogap at $E_{F}$ with the electrical conductivity of a large number of QC and approximant phases. However, up to now we have only considered the averaged $d I / d V$ spectra, where the information about the locally varying fine structure is not present anymore (cf. Fig. 1). Therefore, we will concentrate in the following discussion on the analysis of the single-spectrum fine structure with regard to its spatial variation, which might be taken as an indication of localization.

\section{B. Local and energetic variation of single-spectra $d I / d V$ correlating with topography}

As shown in Fig. 1(b), single STS spectra representing the LDOS at a given position display a variety of fine-structure features. The spatial variation of the LDOS fine structure on the subnanometer level has been investigated by Widmer $e t$ al. on the fivefold and twofold $i$-Al-Pd-Mn surfaces. ${ }^{31,61}$ The very complex surface structures prevented a conclusive assignment of $d I / d V$ fine structures with topography features. However, the simpler surface structure of the (12110) twofold $d$-Al-NiCo, exhibiting at the same time a periodic and a quasiperiodic direction, reveals a clear correlation of spiky features in the LDOS with topography. 
TABLE II. Lists the set point of the $d I / d V$ measurements, the $b / a$ ratio from the $F(V)$ fit of the averaged $d I / d V$ spectra, as well as the literature values of the electrical resistivity.

\begin{tabular}{|c|c|c|c|c|c|c|c|}
\hline Systems & $\begin{array}{l}i \text {-Al-Pd-Mn } \\
\text { Fivefold }\end{array}$ & $\begin{array}{c}i \text {-Al-Pd-Mn } \\
\text { Twofold }\end{array}$ & $\begin{array}{c}p \text {-tenfold } \\
\xi^{\prime}- \\
\text { Al-Pd-Mn }\end{array}$ & $\begin{array}{c}d-\mathrm{Al}-\mathrm{Ni}-\mathrm{Co} \\
\text { Tenfold }\end{array}$ & $\begin{array}{c}d \text {-Al-Ni-Co } \\
\text { Twofold }\end{array}$ & $\begin{array}{l}\text { (100)-Y- } \\
\text { Al-Ni-Co }\end{array}$ & $\begin{array}{c}\mathrm{Al} \\
(111)\end{array}$ \\
\hline \multicolumn{8}{|l|}{ Set point } \\
\hline$U_{T}(V)$ & 0.5 & -1 & 0.7 & 0.5 & 1 & -1 & 1 \\
\hline$I_{T}(\mathrm{nA})$ & 0.2 & 0.15 & 0.25 & 0.2 & 0.6 & 0.2 & 0.25 \\
\hline$R_{T}(\mathrm{G} \Omega)$ & 2.5 & 6.6 & 2.8 & 2.5 & 1.66 & 5 & 4 \\
\hline $\begin{array}{l}\text { Probed surface } \\
\text { area }\left(\mathrm{nm}^{2}\right)\end{array}$ & $10 \times 10$ & $10 \times 10$ & $13 \times 8$ & $30 \times 19.3$ & $10 \times 10$ & $5 \times 5$ & $10 \times 10$ \\
\hline $\begin{array}{l}\text { Number of single } \\
d I / d V \text { spectra }\end{array}$ & 2380 & 2500 & 570 & 260 & 160 & 2500 & 3600 \\
\hline$b / a$ ratio & 7.0 & 5.3 & 5.2 & 10.8 & 7.4 & 6.0 & 1.5 \\
\hline $\begin{array}{l}\text { Electrical } \\
\text { resistivity } \\
(\mu \Omega \mathrm{cm})\end{array}$ & $\begin{array}{c}1350 \\
\text { (Ref. 5) }\end{array}$ & $\begin{array}{c}1350 \\
(\text { Ref. 5) }\end{array}$ & $\begin{array}{c}213 \\
\text { (Ref. 57) }\end{array}$ & $\begin{array}{c}340^{\mathrm{a}} \\
\text { (Ref. 4) }\end{array}$ & $\begin{array}{c}38^{\mathrm{a}} \\
\text { (Ref. 4) }\end{array}$ & $\begin{array}{c}19.5^{\mathrm{b}} \\
\text { (Ref. 10) }\end{array}$ & $\begin{array}{c}0.001 \\
\text { (Refs. } 58 \text { and 59) }\end{array}$ \\
\hline
\end{tabular}

${ }^{\text {a For the tenfold } d-\mathrm{Al}_{77} \mathrm{Ni}_{17} \mathrm{Co}_{6} \text { the electrical resistivity along the quasiperiodic direction and for the twofold-(12110) } d-\mathrm{Al}_{72.9} \mathrm{Ni}_{10.4} \mathrm{Co}_{16.7}}$ surface along the periodic direction is used.

${ }^{\mathrm{b}}$ The mean value of the in-plane measured electrical resistivity $\rho_{c}=29 \mu \Omega \mathrm{cm}$ and $\rho_{b}=10 \mu \Omega \mathrm{cm}$ is used.

${ }^{\mathrm{c}}$ These are the low-temperature values $(2-5 \mathrm{~K})$ according to mentioned literature.

Figure 3(a) displays the topographic STM image of the (12110) twofold $d$-Al-Ni-Co surface. The quasiperiodic axis is vertically and the periodic axis horizontally (with a small tilting) aligned. Along an $0.8-\mathrm{nm}$ periodic column, at the intersection with Fig. 3(b), the $d I / d V$ spectra have been recorded with a spacing of $0.075 \mathrm{~nm}$. The spatially resolved background subtracted [according to Eq. (4)] $d I / d V$ intensity map is shown in Fig. 3(b). The most apparent characteristic is an $0.8-\mathrm{nm}$ modulation in the $d I / d V$ intensity along the periodic axis. Features in the $d I / d V$ spectra with high intensity (peaks) in the positive sample bias range (unoccupied

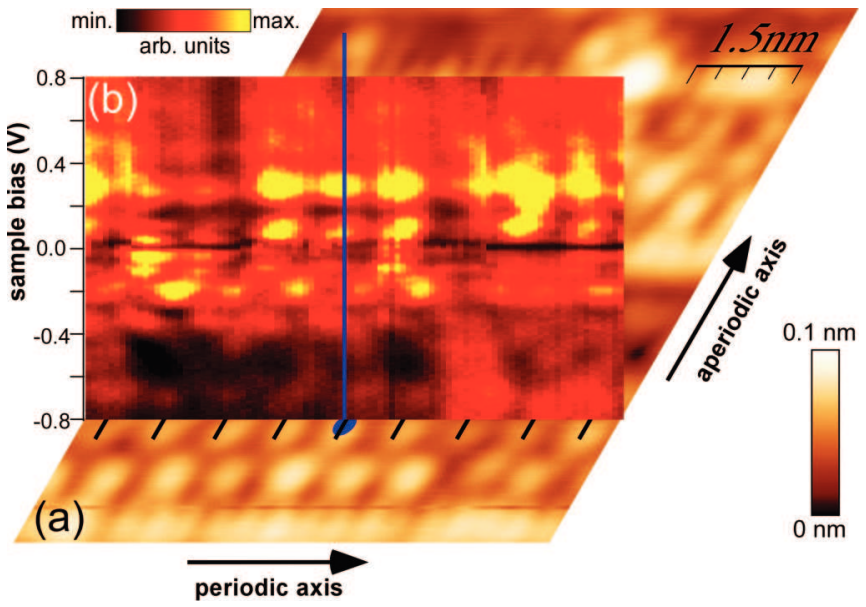

FIG. 3. (Color online) Correlation of peaks and pseudogaps of the differential conductance with topography. (a) $7.5 \times 7.5 \mathrm{~nm}^{2}$ STM image of the twofold (12110) $d$-Al-Ni-Co surface recorded at $5.4 \mathrm{~K}, \Delta z=0.10 \mathrm{~nm}, V_{T}=-0.5 \mathrm{~V}, I_{T}=0.1 \mathrm{nA}$. (b) A spatially resolved background subtracted $d I / d V$ intensity map along an 0.8 -nm periodic columnar structure whereas high intensity is indicated by yellow and low intensity by black color. For more details, see Ref. 12.
LDOS) are in registry with topography protrusions. On the other hand, within the negative sample bias range (occupied LDOS) peaks at -0.2 and $-0.45 \mathrm{~V}$ are shifted by $0.4 \mathrm{~nm}$ and appear at topographic depressions. The characteristic extent of the spectral features is about $0.1 \mathrm{eV}$ in energy and the spatial confinement along the periodic axis is about $0.45 \mathrm{~nm}$. This result clearly depicts that for specific energies, spatially localized electronic states are present and possess a correlation with topographic features. The observation of site-specific localization of electronic states agrees well with the concept of "cluster virtual bound states" described by Trambly de Laissardière et al. ${ }^{13}$ In this concept, valence electronic states are trapped by transition-metal clusters (atomic structures) due to a scattering mechanism. It is found that the more spherical the atomic structures are, the more efficient is the localization of the electronic states and thus sharper spikes in LDOS are emerging. In the following, we are parametrizing the local variations of the fine-structure features and compare it to the electrical resistivity.

\section{Analysis of the variation in the differential conductance and its correlation with the electrical resistivity}

As mentioned above, Trambly de Laissardière et al..$^{13}$ reported that an increase of the electron localization results in a narrowing of the peaks in the DOS. Stronger localizations do not only show sharper peaks, but also an increased peak intensity. ${ }^{13}$ With regard to the here discussed $d I / d V$ spectra, the strength of the localization can be estimated by considering the intensity of the spiky peaks only. For this task, we are considering the deviation of the single $d I / d V(V)$ spectra in percent from the average spectrum of the set of $d I / d V(V)$ spectra measured on a grid of points $\left(x_{i}, y_{i}\right)$ with $i=1, \ldots, N_{s}$. Here, $N_{s}$ denotes the number of spectra measured on a sample and is given in Table I for the different surfaces under consideration. $S\left(V, x_{i}, y_{i}\right)$ for $i=1, \ldots, N_{s}$ describes the 
magnitude of the deviation of specific spectral features from the averaged $d I / d V$ spectrum at each measured grid point for each measured bias voltage $V$ :

$$
S\left(V, x_{i}, y_{i}\right)=\frac{\frac{d I}{d V}\left(V, x_{i}, y_{i}\right)-\frac{d I_{\mathrm{Avg}}}{d V}(V)}{\frac{d I_{\mathrm{Avg}}}{d V}(V)} 100 \%
$$

with

$$
\frac{d I_{\mathrm{Avg}}}{d V}(V)=\frac{1}{N_{s}} \sum_{i=1}^{N_{s}} \frac{d I}{d V}\left(V, x_{i}, y_{i}\right) .
$$

From the $S\left(V, x_{i}, y_{i}\right)$ data set, we compute for each bias voltage $V$ the histogram of $S$ over all measured positions $\left(x_{i}, y_{i}\right)$ yielding the two-dimensional frequency distribution $\operatorname{FD}(V, S)$, which is displayed color coded in Fig. 4 for the different surfaces. The value of $S(V, x, y)$ averaged over all grid points is by definition $0 \%$ and is indicated by the central red line. The corresponding standard deviations are shown by the adjacent red lines. The histogram plots in Fig. 4 reveal a strong spatial variation of the single spectra in the whole bias range for the QC systems, where the deviation can be significantly larger than $100 \%$. This is especially apparent for the fivefold Al-Pd-Mn surface. On the other hand, the approximant surfaces show a reduced variation in the single spectra and almost vanishes for the $\mathrm{Al}(111)$ surface. Please note that there is a systematically reduced variation bandwidth on the set-point side, which originates from the constant current measurement mode.

In order to parametrize the spectral variations for a given system, as displayed in Fig. 4, we take the value of the standard deviation averaged over the full bias range. This procedure yields a single parameter $\hat{S}$, which has no direct physical significance, but serves as a measure of the magnitude of the spatial spectra variations. In this sense, $\hat{S}$ can be regarded to reflect the localization of electronic states and we can attempt a comparison with values of the electrical resistivity. ${ }^{16,42}$ In Fig. 5, $\hat{\mathrm{S}}$ of all investigated surfaces is plotted versus the square root of the electrical resistivity [literature values, which have been measured at low temperatures $(\sim 4 \mathrm{~K})$ (see Table II)]. The plot of the nanoscopic $\hat{S}$ parameter in Fig. 5 reveals a clear linear dependence on the square root of the reported electrical resistivity, which is a macroscopic physical quantity. The line fit represented by the black dashed line in Fig. 5 corresponds to $\hat{S}=9.8+0.86 \sqrt{\rho}$. The sequence of $\hat{S}$ for the different systems follows an intuitive order of structural complexity. This is expressed by the reduction of either the number of quasiperiodic dimensions in the systems or of the number of atoms per unit cell. Namely, the highest $\hat{S}$ are found for the fivefold icosahedral Al-Pd-Mn (bulk: quasiperiodic in all three physical dimensions) and the tenfold decagonal Al-Ni-Co (bulk: two quasiperiodic and one periodic dimension) QC surfaces. The sequence is continued by $\hat{\mathrm{S}}$ of the pseudo tenfold $\xi^{\prime}$-Al-Pd-Mn (320 atoms per unit cell), the twofold $d$-Al-Ni-Co (surface: spanned by periodic and quasiperiodic axes), the (100) Y-Al-Ni-Co (32 atoms per unit cell) approximant surfaces, and ends with the $\mathrm{Al}(111)$ surface. Additionally, we investigated the (001) surface of $\mu-\mathrm{Al}_{4} \mathrm{Mn}$ with 563 atoms per unit cell and an $\hat{\mathrm{S}}$ value of 35.3 was determined, which situates this surface between the
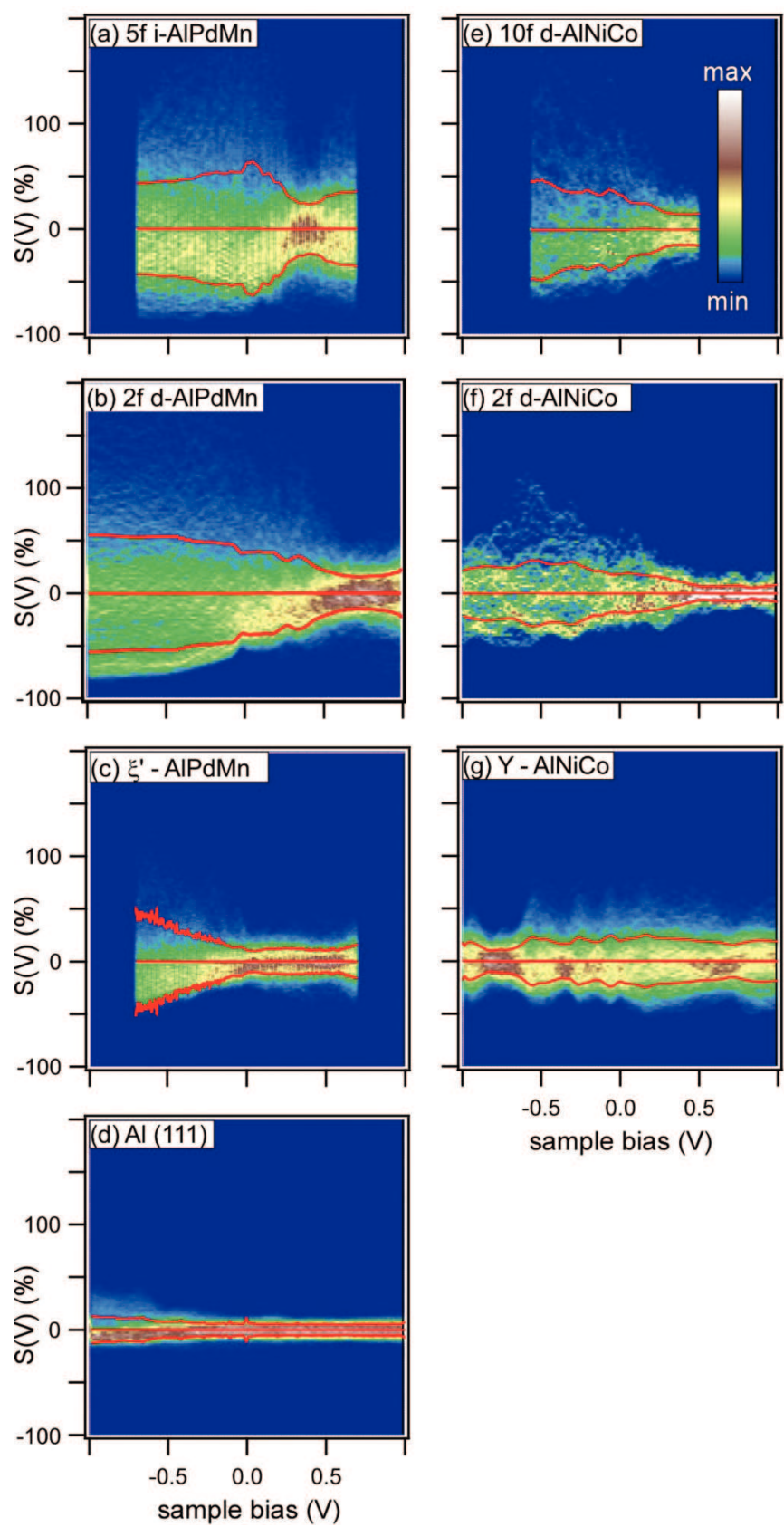

sample bias $(\mathrm{V})$

FIG. 4. (Color online) Scatter plot images of the difference in the differential conductance between a single spectrum and the averaged spectrum $S(V)$ vs sample bias. For details, see text.

$i$-AlPdMn and the tenfold $d$-AlNiCo surfaces. Due to lack of reference data, we did not include these measurements in Fig. 5. However, from the linear dependence shown in Fig. 5, we derived a $\sqrt{\rho_{4 \mathrm{~K}}(\mu \Omega \mathrm{cm})}$ value around 30 corresponding to a resistivity of $888 \mu \Omega \mathrm{cm}$. This value is considerably higher than what is reported in literature ${ }^{62}$ for the resistivity measured perpendicular to the $(001)$ direction $(356 \mu \Omega \mathrm{cm})$. The reason for such a large difference may originate from the structural icosahedral chains aligned along the [100] crystal direction of $\mu-\mathrm{Al}_{4} \mathrm{Mn} .{ }^{63}$

One apparent feature of this line fit is an offset of $\sim 10 \%$ in $\hat{\mathrm{S}}$. The analysis on the origin of this offset is performed by taking a closer look on the $d I / d V$ spectra of the $\mathrm{Al}(111)$ 


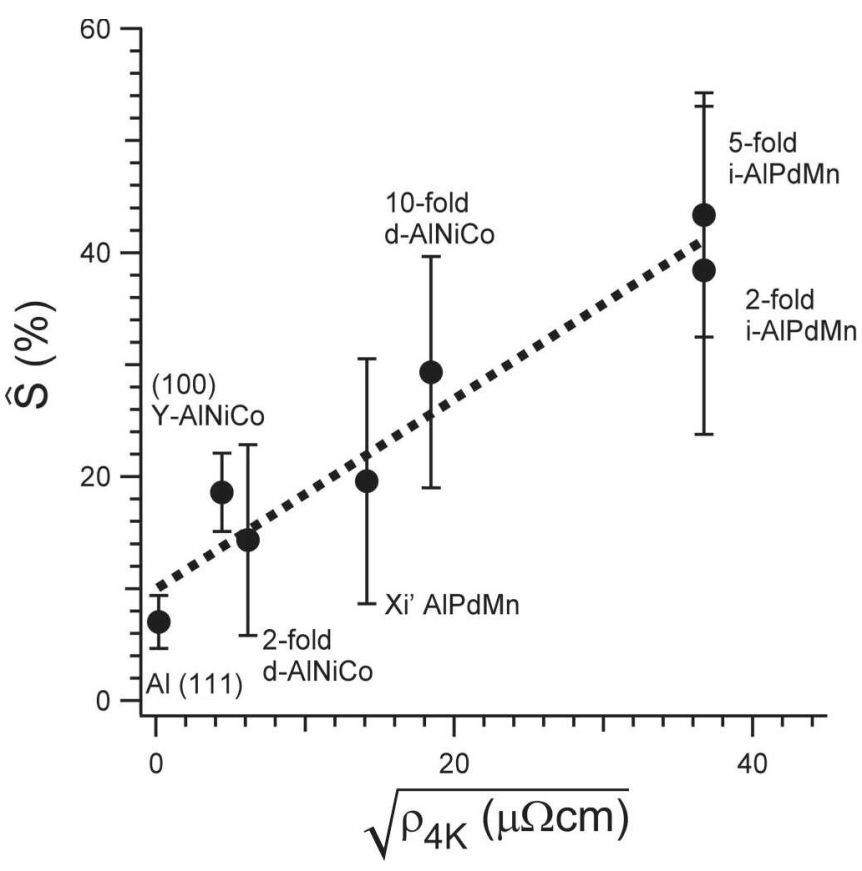

FIG. 5. Standard deviation of the difference between single spectra and average spectrum of the differential conductance vs the square root of the literature values of the electrical resistivity measured at $4 \mathrm{~K}$ is presented (for more details, see text).

surface. As the atomic structure of $\mathrm{Al}(111)$ consists of indistinguishable sites only, in principle no local variation in $d I / d V$ spectra and $\hat{S}=0 \%$ is expected. Nevertheless, we observe a value of $\hat{S}=7.0 \%$ for $\mathrm{Al}(111)$. Inspection of the single spectra in the grid shows that they differ only by a proportionality factor, which can be related to slightly different tip-sample distances. A difference of $7 \%$ in tunneling current corresponds to a tip-sample distance variation of roughly $2 \mathrm{pm}$, which is the typical mechanical noise in our LT-STM system.

Our analysis considers $\hat{S}$ over the full applied sample bias range, even though the states involved in electronic transport are very close to $E_{F}$. This consideration can by justified by the following reasons. First, measuring $\hat{S}$ very close to $E_{F}$ is hindered by particular tunneling transport characteristics occurring at zero bias such as, e.g., Coulomb blockade. ${ }^{36}$

Second, the localization effects in the crystals studied here, namely, Brillouin zone interaction and orbital hybridization, are not directly related to $E_{F}$ in the sense that they can lead to opening of gaps or pseudogaps where an electron band crosses the zone boundary associated with a strongly scattering crystal plane or where two hybridizing bands cross in energy. ${ }^{17,21,64,65}$ Therefore, they can express themselves in a much larger energy region around $E_{F}$ than the few meV from which the electron contributing to the current in transport experiments originates from. This justifies our parametrization scheme of $\hat{S}$ that considers states within the range of $+/-1 \mathrm{eV}$ around $E_{F}$.

Finally, we like to discuss the dependency $\hat{\mathrm{S}}$ with $\sqrt{\rho}$. Two theoretical expressions for the electrical resistivity of QC and approximants have been derived: one by Mizutani ${ }^{16}$ and the other by Trambly de Laissardière et al. ${ }^{42}$ The electron localization is incorporated differently in both expressions:
Mizutani ${ }^{16}$ used the hopping integral parameter $I_{H}$, whereas Trambly de Laissardière et $a l .{ }^{42}$ considered the velocity of the charge-carrier wave packets $V_{P}$. Both parameters are inversely proportional to the localization, which means they decrease for an increasing degree of localization. The square root of the electrical resistivity is thus inversely proportional to the corresponding parameter. We find the same dependence with regard to the $\hat{S}$ parameter in the sense that

$$
\sqrt{\rho} \propto \frac{1}{I_{H}} \propto \frac{1}{V_{P}} \propto \hat{S} .
$$

This can be taken as confirmation that our parametrization of the local variability of the $d I / d V$ spectra around $E_{F}$ can indeed be taken as a measure of localization.

\section{SUMMARY AND CONCLUSION}

We have performed a STS study in the Al-Ni-Co and the Al-Pd-Mn systems to explore the origin of the remarkably high electrical resistivities of QC and approximants. A normalization method for STS experiments has been implemented to reveal the sample DOS for systems possessing a wide pseudogap at $E_{F}$. This method has been successfully validated for the Al-Ni-Co and the Al-Pd-Mn systems. The shape of the pseudogap in the DOS has been analyzed by the curvature to offset ratio $(b / a)$. The $b / a$ value indicates the depth and width of the pseudogap. Between different systems, the trend of the $b / a$ values agrees with results obtained by UPS measurements. We observed a stronger reduction in the DOS for QC compared to approximant systems, but no correlation could be found between the depths of the pseudogaps ( $b / a$ parameter) and the reported electrical resistivities.

However, we have revealed a clear correlation of the reported electrical resistivity, which is a macroscopic bulk property, with a parameter derived from STS grid measurements of the different systems with subnanometer resolution. This parameter $\hat{\mathrm{S}}$ quantifies the spatial variability of the fine-structure features in $d I / d V$ spectra. It can be interpreted as a measure for the magnitude of the electron localization of a given system. In agreement with previous theoretical work, we observe a square-root dependence of the electrical resistivity with the corresponding $\hat{\mathrm{S}}$ parameter. Our findings are consistent with the concept of cluster virtual bound states and the transport of slow charge carriers as reported by Trambly de Laissardière et al. ${ }^{13,42}$ This result indicates that the interaction between valence electrons and the local complex local atomic arrangement plays a significant role on the macroscopic measurable electrical resistivity.

\section{ACKNOWLEDGMENTS}

We thank M. Feuerbacher, M. Heggen, and P. Gille for providing us with high-quality single crystals. The Swiss National Foundation (Contract No. SNF 200021-112333/1), the European Network of Excellence (NoE): "Complex Metallic Alloys" CMA (Contract No. E 500140) and the EU-FP7: "appliCMA" project (Grant Agreement No. NMP3-SL-2008214407) are acknowledged. 
${ }^{1}$ D. Shechtman, I. Blech, D. Gratias, and J. W. Cahn, Phys. Rev. Lett. 53, 1951 (1984).

${ }^{2}$ W. Steurer and S. Deloudi, Acta Crystallogr., Sect. A 64, 1 (2008).

${ }^{3}$ Z. M. Stadnik, in Physical Properties of Quasicrystals, edited by Z. M. Stadnik, Vol. 126 (Springer, Berlin, 1999), p. 257.

${ }^{4}$ S. Martin, A. F. Hebard, A. R. Kortan, and F. A. Thiel, Phys. Rev. Lett. 67, 719 (1991).

${ }^{5}$ A. L. Pope, T. M. Tritt, M. A. Chernikov, and M. Feuerbacher, Appl. Phys. Lett. 75, 1854 (1999).

${ }^{6}$ J. Y. Park, D. F. Ogletree, M. Salmeron, R. A. Ribeiro, P. C. Canfield, C. J. Jenks, and P. A. Thiel, Science 309, 1354 (2005).

${ }^{7}$ Physical Properties of Quasicrystals, edited by Z. M. Stadnik (Springer, Berlin, 1999).

${ }^{8}$ S. Deloudi, M. Kobas, and W. Steurer, Philos. Mag. 86, 581 (2006).

${ }^{9}$ M. Komelj, J. Ivkov, A. Smontara, P. Gille, P. Jeglic, and J. Dolinsek, Solid State Commun. 149, 515 (2009).

${ }^{10}$ A. Smontara, I. Smiljanic, J. Ivkov, D. Stanic, O. S. Barisic, Z. Jaglicic, P. Gille, M. Komelj, P. Jeglic, M. Bobnar, and J. Dolinsek, Phys. Rev. B 78, 104204 (2008).

${ }^{11}$ A. Smontara, D. Stanic, I. Smiljanic, J. Dolinsek, and P. Gille, Z. Kristallogr. 224, 56 (2009).

${ }^{12}$ R. Mäder, R. Widmer, P. Gröning, P. Ruffieux, W. Steurer, and O. Gröning, New J. Phys. 12, 073043 (2010).

${ }^{13}$ G. T. de Laissardiere, D. Nguyen-Manh, and D. Mayou, Prog. Mater. Sci. 50, 679 (2005).

${ }^{14}$ Z. M. Stadnik, D. Purdie, M. Garnier, Y. Baer, A.-P. Tsai, A. Inoue, K. Edagawa, S. Takeuchi, and K. H. J. Buschow, Phys. Rev. B 55, 10938 (1997).

${ }^{15}$ K. Soda, K. Nozawa, Y. Yanagida, K. Morita, U. Mizutani, Y. Yokoyama, R. Note, A. Inoue, H. Ishii, Y. Tezuka, and S. Shin, J. Electron Spectrosc. Relat. Phenom. 88, 415 (1998).

${ }^{16}$ U. Mizutani, J. Phys.: Condens. Matter 10, 4609 (1998).

${ }^{17}$ G. T. de Laissardiere, Z. Kristallogr. 224, 123 (2009).

${ }^{18}$ E. Belin-Ferre, J. Non-Cryst. Solids 334, 323 (2004).

${ }^{19}$ A. K. Shukla, C. Biswas, R. S. Dhaka, S. C. Das, P. Kruger, and S. R. Barman, Phys. Rev. B 77, 195103 (2008).

${ }^{20}$ J. Hafner and M. Krajci, Phys. Rev. Lett. 68, 2321 (1992).

${ }^{21}$ G. Trambly de Laissardière and T. Fujiwara, Phys. Rev. B 50, 9843 (1994).

${ }^{22} \mathrm{U}$. Mizutani, Introduction to the Electron Theory of Metals (Cambridge University Press, Cambridge, 2001).

${ }^{23}$ M. Krajci and J. Hafner, Phys. Rev. B 71, 054202 (2005).

${ }^{24}$ M. Krajci, J. Hafner, and M. Mihalkovic, Phys. Rev. B 73, 134203 (2006).

${ }^{25}$ R. Tamura, Y. Murao, S. Takeuchi, T. Kiss, T. Yokoya, and S. Shin, Phys. Rev. B 65, 224207 (2002).

${ }^{26}$ U. Mizutani, T. Takeuchi, and H. Sato, J. Non-Cryst. Solids 334, 331 (2004).

${ }^{27}$ D. Naumovic, P. Aebi, L. Schlapbach, C. Beeli, K. Kunze, T. A. Lograsso, and D. W. Delaney, Phys. Rev. Lett. 87, 195506 (2001).

${ }^{28}$ D. Naumovic, P. Aebi, L. Schlapbach, C. Beeli, T. A. Lograsso, and D. W. Delaney, Phys. Rev. B 60, R16330 (1999).

${ }^{29}$ U. Mizutani, M. Inukai, H. Sato, and E. Zijlstra, Chem. Soc. Rev. 41, 6799 (2012).

${ }^{30}$ E. S. Zijlstra and T. Janssen, Europhys. Lett. 52, 578 (2000).
${ }^{31}$ R. Widmer, P. Gröning, M. Feuerbacher, and O. Gröning, Phys. Rev. B 79, 104202 (2009).

${ }^{32}$ Z. M. Stadnik, D. Purdie, Y. Baer, and T. A. Lograsso, Phys. Rev. B 64, 214202 (2001).

${ }^{33}$ R. Escudero, J. C. Lasjaunias, Y. Calvayrac, and M. Boudard, J. Phys.: Condens. Matter 11, 383 (1999).

${ }^{34}$ J. T. Okada, T. Ekino, Y. Yokoyama, T. Takasaki, Y. Watanabe, and S. Nanao, J. Phys. Soc. Jpn. 76, 033707 (2007).

${ }^{35}$ D. N. Davydov, D. Mayou, C. Berger, and A. G. M. Jansen, Int. J. Mod. Phys. B 12, 503 (1998).

${ }^{36}$ J. Delahaye, T. Schaub, C. Berger, and Y. Calvayrac, Phys. Rev. B 67, 214201 (2003).

${ }^{37}$ R. Widmer, O. Groening, P. Ruffieux, and P. Groening, Philos. Mag. 86, 781 (2006).

${ }^{38}$ T. Fujiwara, S. Yamamoto, and G. Trambly de Laissardière, Phys. Rev. Lett. 71, 4166 (1993).

${ }^{39}$ E. S. Zijlstra and T. Janssen, Phys. Rev. B 61, 3377 (2000).

${ }^{40}$ J. Delahaye, C. Gignoux, T. Schaub, C. Berger, T. Grenet, A. Sulpice, J. J. Prejean, and J. C. Lasjaunias, J. Non-Cryst. Solids 252, 878 (1998).

${ }^{41}$ T. Schaub, J. Delahaye, C. Gignoux, C. Berger, G. Fourcaudot, F. Giroud, T. Grenet, and A. G. M. Jansen, J. Non-Cryst. Solids 252, 874 (1998).

${ }^{42}$ G. Trambly de Laissardière, J.-P. Julien, and D. Mayou, Phys. Rev. Lett. 97, 026601 (2006).

${ }^{43}$ R. Mäder, R. Widmer, B. Bauer, P. Gille, P. Gröning, W. Steurer, and O. Gröning, Phys. Rev. B 81, 064201 (2010).

${ }^{44}$ V. Fournee, A. R. Ross, T. A. Lograsso, J. W. Anderegg, C. Dong, M. Kramer, I. R. Fisher, P. C. Canfield, and P. A. Thiel, Phys. Rev. B 66, 165423 (2002).

${ }^{45}$ R. Widmer, R. Maeder, M. Heggen, M. Feuerbacher, and O. Gröning, Philos. Mag. 88, 2095 (2008).

${ }^{46}$ R. Mäder, R. Widmer, P. Gröning, S. Deloudi, W. Steurer, M. Heggen, P. Schall, M. Feuerbacher, and O. Gröning, Phys. Rev. B 80, 035433 (2009).

${ }^{47}$ H. W. Sheng, W. K. Luo, F. M. Alamgir, J. M. Bai, and E. Ma, Nature (London) 439, 419 (2006).

${ }^{48}$ M. Schmid, W. Hebenstreit, P. Varga, and S. Crampin, Phys. Rev. Lett. 76, 2298 (1996).

${ }^{49}$ J. Wintterlin, J. Wiechers, H. Brune, T. Gritsch, H. Hofer, and R. J. Behm, Phys. Rev. Lett. 62, 59 (1989).

${ }^{50}$ J. Tersoff and D. R. Hamann, Phys. Rev. Lett. 50, 1998 (1983).

${ }^{51}$ R. M. Feenstra, J. A. Stroscio, and A. P. Fein, Surf. Sci. 181, 295 (1987).

${ }^{52}$ R. J. Hamers, Annu. Rev. Phys. Chem. 40, 531 (1989).

${ }^{53}$ M. Prietsch, A. Samsavar, and R. Ludeke, Phys. Rev. B 43, 11850 (1991).

${ }^{54}$ V. A. Ukraintsev, Phys. Rev. B 53, 11176 (1996).

${ }^{55}$ T. Suzuki, H. R. Sharma, T. Nishimura, M. Shimoda, Y. Yamauchi, and A. P. Tsai, Phys. Rev. B 72, 115427 (2005).

${ }^{56}$ G. Kasner, Z. Papadopolos, P. Kramer, and D. E. Burgler, Phys. Rev. B 60, 3899 (1999).

${ }^{57}$ J. Dolinsek, P. Jeglic, P. J. McGuiness, Z. Jaglicic, A. Bilusic, Z. Bihar, A. Smontara, C. V. Landauro, M. Feuerbacher, B. Grushko, and K. Urban, Phys. Rev. B 72, 064208 (2005). 
${ }^{58}$ A. v. Bassewitz and E. N. Mitchell, Phys. Rev. 182, 712 (1969).

${ }^{59} \mathrm{CRC}$ Handbook of Chemistry and Physics, edited by D. R. Lide (CRC Press, Boca Raton, FL, 2005).

${ }^{60}$ T. Klein, O. G. Symko, D. N. Davydov, and A. G. M. Jansen, Phys. Rev. Lett. 74, 3656 (1995).

${ }^{61}$ R. Widmer, O. Gröning, P. Ruffieux, and P. Gröning, Philos. Mag. 86, 781 (2006).
${ }^{62}$ M. Wencka, S. Jazbec, Z. Jaglicic, S. Vrtnik, M. Feuerbacher, M. Heggen, S. Roitsch, and J. Dolinsek, Philos. Mag. 91, 2756 (2011).

${ }^{63}$ C. B. Shoemaker, Philos. Mag. B 67, 869 (1993).

${ }^{64}$ U. Mizutani, T. Takeuchi, and H. Sato, Prog. Mater Sci. 49, 227 (2004).

${ }^{65}$ T. Fujiwara, Mater. Sci. Eng. B 19, 77 (1993). 